\title{
Low Cost Embedded System for Voltage Sag Analysis
}

\author{
Aswathy M \\ PG Scholar \\ Electrical and Electronics Department \\ Amrita Vishwa Vidyapeetham \\ Coimbatore,India
}

\author{
R Jayabarathi \\ Associate Professor \\ Electrical and Electronics Department \\ Amrita Vishwa Vidyapeetham \\ Coimbatore,India
}

\begin{abstract}
This paper describes the development of low cost embedded system for detecting and analysing the voltage sags in the ac supply. The proposed system have the capabilities of real time acquisition of voltage signal using an Embedded Controller and display its parameters in a Graphical User Interface in the Central Server (PC).The features of the signal are extracted using Discrete Wavelet Transform with Multi Resolution Analysis. The voltage sags are created in an experimental setup using voltage injection method. The real time voltages are sensed by Hall Effect Transducer and digitalised by mbed microcontroller (LPC1768 ARM Cortex-M3).The real time samples are then serially transmitted to the Central Server. A standalone application is developed in Central Server using Visual C Sharp for real time visualisation, processing and analysis of the signal.
\end{abstract}

\section{General Terms}

Discrete Wavelet Transforms, Power Quality, Voltage Sag

\section{Keywords}

Power Quality (PQ), Discrete Wavelet Transform (DWT), Multi Resolution Analysis (MRA), Graphical User Interface (GUI), rms (root mean square).

\section{INTRODUCTION}

Power Quality is an important concern for both electric utilities and end users. The PQ disturbances affect the operation of sensitive equipments such as programmable logic controllers, adjustable speed drives, switched mode power supplies etc. The power quality problems may result in the deviation of voltage and current and thus mal function the equipment. The common power quality disturbances are voltage sag, voltage swell, harmonics, flickers, glitches, transients, notches etc

Voltage sags are one of the most critical disruptions in the ac supply. They are short time voltage variations which disrupts the working of sensitive equipments. The IEEE 1159 Standard defines voltage sag as the reduction in rms voltage between 0.1p.u-0.9p.u for a small duration. Therefore early detection and analysis of such PQ disturbances are essential for improving the quality of power and enhancing the productivity of the system.

The traditional instruments such as harmonic analyzer, flicker meter, energy monitors do not possess the capability to locally analyze, interpret, and determine what is happening in the power system. In this scenario, the virtual instruments play a very important role [2]. The automatic recognition of Power Quality disturbances can be effectively done using signal processing techniques. All these years, researchers have used different methods to measure and classify the power quality disturbances and various protocols to control the system [1][4].

The signal processing tools plays an important role in detecting and classifying the different power quality disturbances [4].The different signal processing techniques are Fourier Transform (FT), Short Time Fourier Transform (STFT), Hilbert Transform, Wavelet Transform, Kalman filtering etc. Many researchers have been carried out several PQ studies in the area of Wavelets in order to explore its potential for analysis of signals.

Wavelet analysis is an advanced mathematical signal processing tool used for extracting the features of the signal in time and frequency domain. Unlike Fourier Transforms, Wavelet transform can analyze the non stationary signals. The majority of PQ disturbances occurs for a short time and non repetitive. The main processes of Wavelet transforms are decomposition and reconstruction. Wavelets uses short duration waveforms and it has the capability to adapt the window size for analysis. The Wavelet transforms are used for the detection and identification of fluctuations in power system [5]-[9]. Wavelets with ANN, Fuzzy logic are used to classify the disturbances based on energy, entropy, rms value etc [10-13]. The Wavelet transform (WT) can also be used to determine the total harmonic distortion and harmonics in different level of decomposition are determined [14].

The objective of this paper is to analyze the voltage sag created in the ac supply using DWT-MRA, plot the real time voltage signal and display its duration and magnitude in the application created in Central Server. This paper is organized as follows: The section II gives a brief description of voltage sag and its classification.The section III presents the Wavelet transform study. The section IV gives the system overview of the proposed system. The section $\mathrm{V}$ elaborates the experimental setup of the voltage sag creation. Software design is explained in Section VI. Hardware results are presented in Section VII. The conclusion and future scope of the work is listed in Section VIII.

\section{VOLTAGE SAG STUDY}

Voltage sag or dip is a short duration decrease between $10 \%-90 \%$ of the rms voltage, lasting from 0.5 cycles to 1 minute in duration. A $10 \%$ sag is considered an event during which the rms voltage decreased by 0.1 p.u. If the voltage decreases longer than 1 minute, such voltage variation is under voltage.

Generally, voltage sag is divided into three classes based on the duration of the occurrences. The classes are instantaneous sag, momentary sag and temporary sag. Voltage sag is caused by starting of induction motor, short circuits in the power supply, three phase faults occurred .Fig.1 shows the voltage 
sag event occurred at the $4^{\text {th }}$ cycle and continues until the $6^{\text {th }}$ cycle by a reduction of 0.5 p.u.Table 1 gives the voltage sag classifications based on its duration.

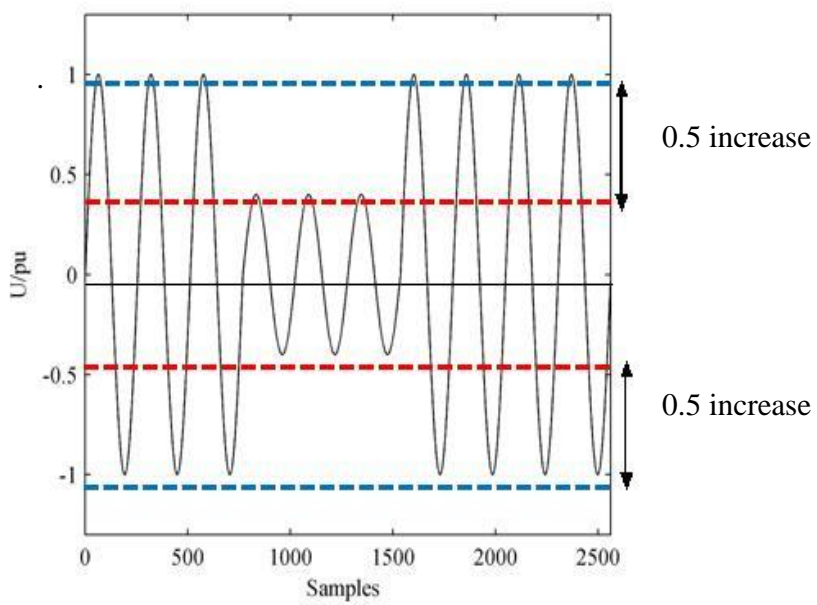

Fig 1: Voltage Sag

Table 1. Voltage Sag Classification

\begin{tabular}{|c|c|c|c|}
\hline Categories & Types & $\begin{array}{c}\text { Typical } \\
\text { Duration }\end{array}$ & $\begin{array}{c}\text { Typical } \\
\text { Magnitude }\end{array}$ \\
\hline Instantaneous & Sag & $0.5-30$ cycles & $0.1-0.9$ p.u \\
\hline Momentary & Sag & $0.5-30$ sec & $0.1-0.9$ p.u \\
\hline Temporary & Sag & 3 sec-1 min & $0.1-0.9$ p.u \\
\hline
\end{tabular}

\section{WAVELET TRANSFORMS}

Wavelets are mathematical functions transform the signal into different frequency components. It is having zero average value and defined over a finite interval. Wavelets are functions that wave above and below the $\mathrm{x}$ axis.Fig. 2 shows a sinusoid and a Wavelet.

Wavelets can represent any function as a sum of basis functions. These basis functions are derived from Mother Wavelet. Discrete Wavelet Transform and Multi Resolution Analysis (MRA) provide a short window for high frequency components and long window for low frequency components and hence provide an excellent time frequency resolution.

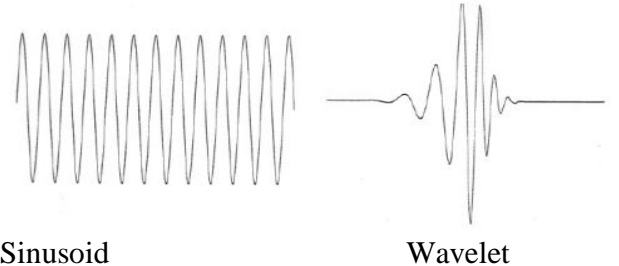

Fig 2: Sinusoid and Wavelet

In Wavelet analysis, the input signal is compared with the Wavelet function to obtain a set of coefficients that represent how these two signals match. The computation of these coefficients is performed using the Continuous WT (CWT).
$\operatorname{CWT}(\mathrm{a}, \mathrm{b})=\frac{1}{\sqrt{ } a} \int_{-\infty}^{\infty} x(t) g *\left(\frac{t-b}{a}\right) d t$

where $x(t)$ is the input signal, $g(t)$ is the mother Wavelet function, and $a$ and $b$ are the scale and the translation factors. Coefficients $\operatorname{CWT}(a, b)$ are the Wavelet representation of a signal $x(t)$ with respect to a Wavelet function $g(t)$.The discrete WT (DWT) is the digital representation of the CWT, and it is defined as

$\operatorname{DWT}(\mathrm{m}, \mathrm{k})=\frac{1}{\sqrt{ } a} \sum_{n} x(n) g *\left(\frac{k-b}{a}\right)$

where $k$ refers to a sample in the input signal and the scale and the translation factors $a$ and $b$ are functions of integer parameters $m$ and $n\left(a=a m_{0}\right.$ and $\left.b=n b_{0} a m_{0}\right)$. Adequately selecting these two parameters, a dyadic decomposition of the time frequency domain is obtained. This scaling produces a logarithmic decomposition of the frequency spectrum.

DWT using a multistage filter bank, as is shown in Fig. 3(a) for a three-level filter bank, with the Wavelet function and its dual as the low-pass (LP) and high-pass (HP) filters, respectively. The output of the HP filter gives the highfrequency components of the signal (the detail version), whereas the output of the LP filter gives the low frequency components (the approximation version). Down sampling by two at the output of the filters $(\downarrow 2)$ scales the Wavelet by two for the next stage.

The output of the LP filter is decomposed again to obtain more details of the signal and so on. At each level of the multistage filter bank in Fig.3(a), the frequency components of the signal are split in two parts, performing the logarithmic decomposition shown in Fig.3(b), where $f s$ is the sampling frequency.
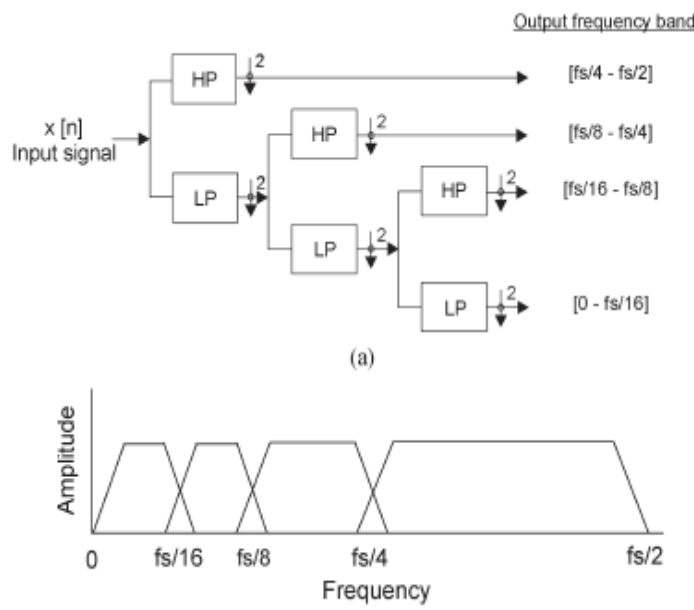

(b)

Fig 3(a): Three-level DWT multistage filter bank and Fig 3(b): logarithmic frequency decomposition 


\section{SYSTEM OVERVIEW}

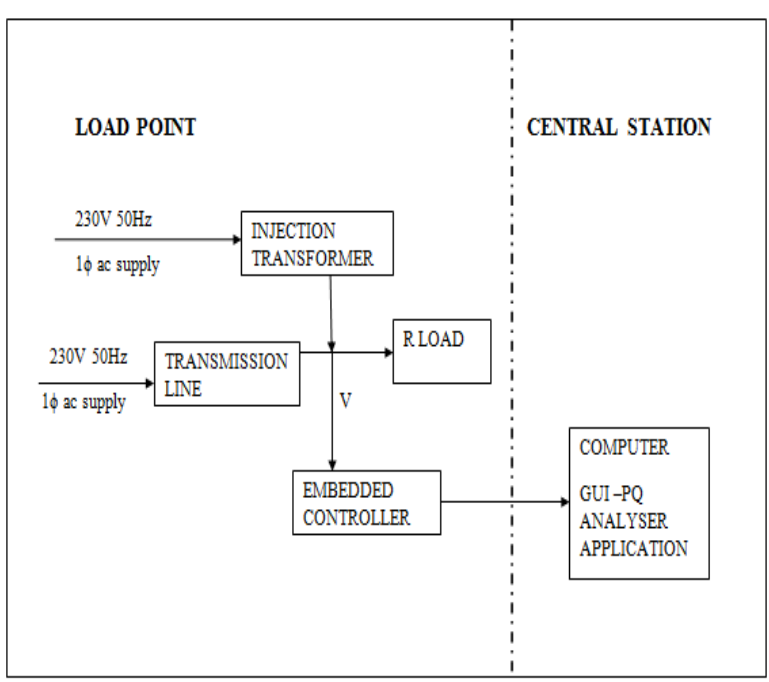

Fig 4:Block diagram for proposed system

The disturbance in the AC Supply is monitored using the proposed system. The voltage is sensed by voltage sensor which is shifted using the level shifter circuit and given to the Embedded Microcontroller. The real time samples are then transmitted to the central server (PC) either by wired or wireless communication. A GUI is created using Visual C Sharp 2010 which runs the MATLAB application for real time signal processing of data and thus the parameters are extracted. The main block diagram is shown in Fig.4.

First the basic circuit for the voltage measurement is setup with the transmission line and $\mathrm{R}$ load and the measurement was done using ammeter and voltmeter. In order to transmit the voltage signals to the controller, the voltage is transformed into appropriate voltages using Voltage Transducer.

The voltage obtained from the voltage transducer are bipolar signals i.e., sinusoidal signals. In order to convert it to unipolar the clamping circuit is provided, which clamps the signals according to the required value as the mbed processor has unipolar ADC. Non-inverting mode adder is used as the clamper circuit. The clamping of the signal is done in order to provide it as the input to the microcontroller. The signals given to the controller should be sampled and transmitted to the Central Station either by wired or wireless communication.

\section{EXPERIMENTAL SETUP FOR VOLTAGE SAG CREATION}

The input supply of $135 \mathrm{~V}, 50 \mathrm{~Hz}, 1 \phi$ ac is given to the transmission line and $230 \mathrm{~V}, 50 \mathrm{~Hz}, 1 \phi$ ac supply is given to the injection transformer $(230 / 30 \mathrm{~V})$. The connections are made as per the Fig.5.

The voltage sag/swell is created using an injection transformer connected in series with the transmission line. When the supply is switched on and relay is turned off, normal voltage is seen across the load.

As the relay operates, voltage is injected into the circuit and gives an increase/decrease of voltage. It is clearly dependent on the transformer polarities. Either sag/swell can be obtained by interchanging the transformer connections. The load voltage is given to the potential transducer and then to the level shifter circuit so as to obtain a voltage compatible to the controller.

\section{SOFTWARE DESIGN}

The Discrete Wavelet Transform with MRA is the signal processing tool used for the detection of distortions in the signal. The choice of mother Wavelet plays a significant role in detecting and localizing different kind of signal changes. The choice depends on the nature and kind of application.

For the detection of low amplitude, short duration, fast decaying and oscillating type signals, Daubechies Wavelets (db4, db6, db10) are used. Those Wavelets have a smoothness property which localises the non stationary data. In this work db6 with 6 level Wavelet decomposition is used.Fig.6 presents the simulation results of a voltage sag signal which occurs for 120 ms duration subjected with DWT-MRA.

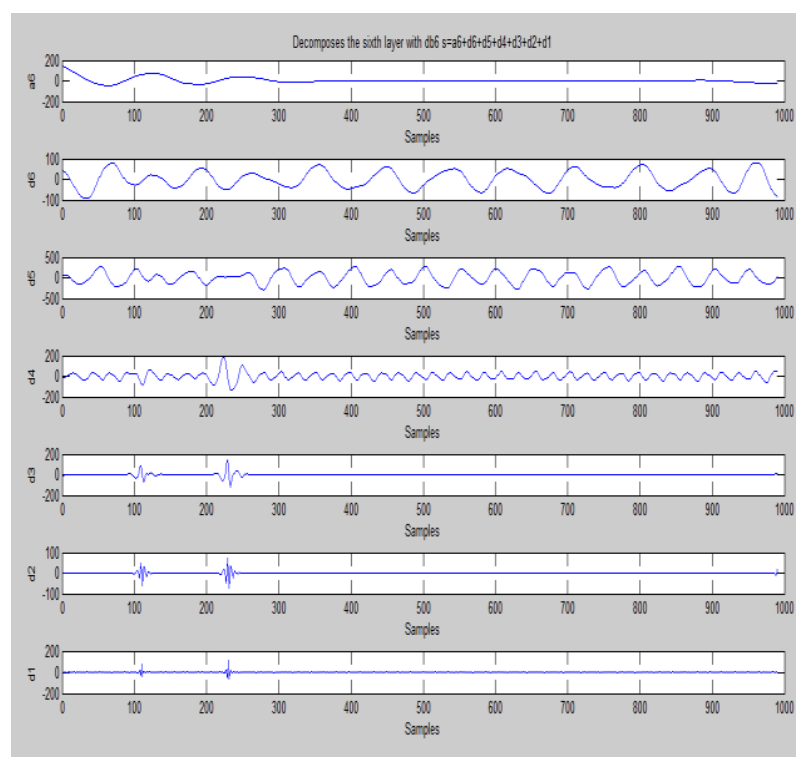

Fig 6: Voltage Sag DWT-MRA Decomposition Diagram 


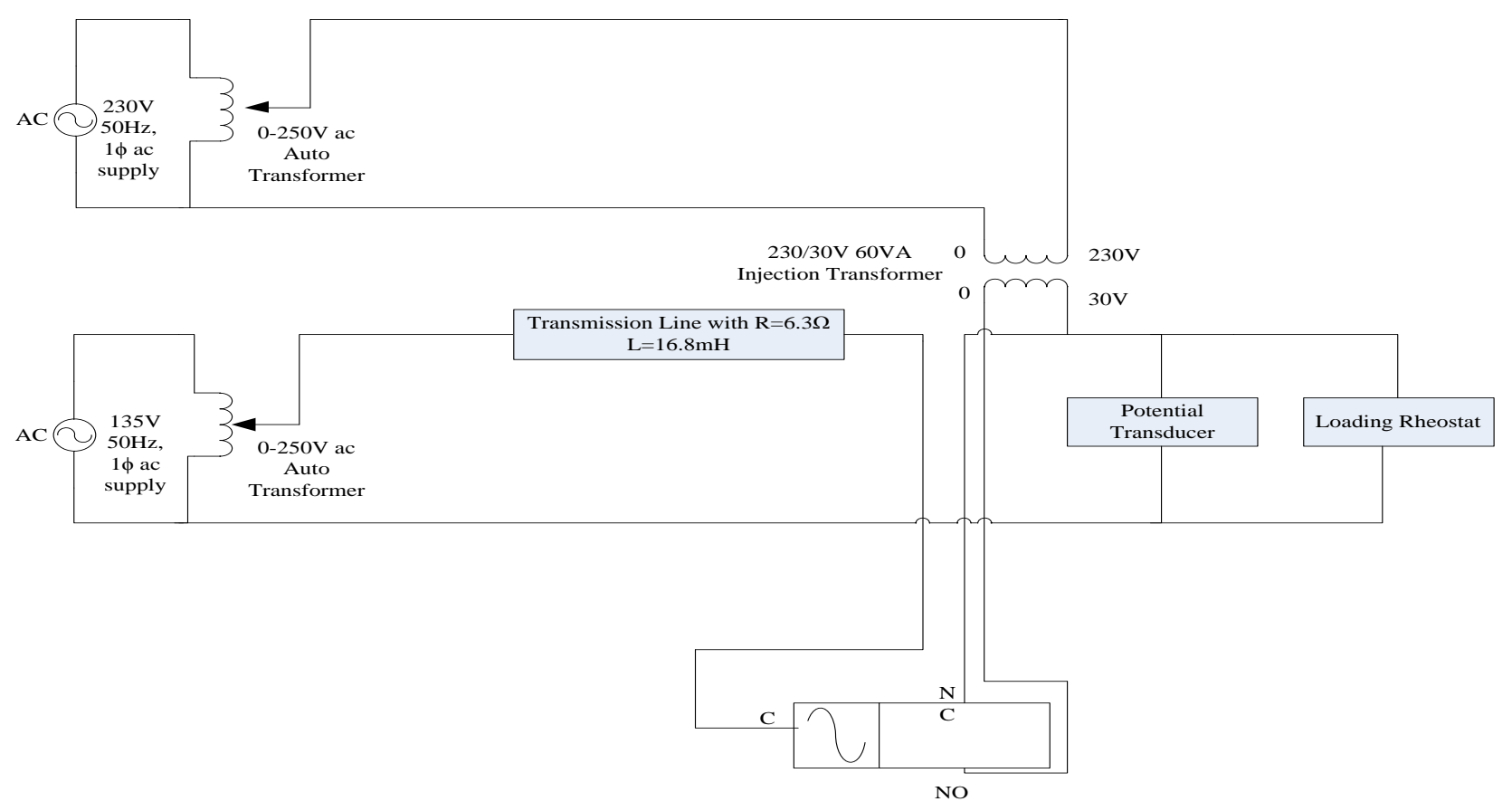

5A 12 V 240Vac Relay

Fig 5: CircuitDiagram

The real time voltage signal is sampled at $1 \mathrm{kHz}$ by mbed LPC1768 microcontroller. The real time voltage samples from controller are automatically saved in GUI as CSV format. Those samples are subjected with DWT-MRA db6 Wavelet. After the Wavelet decomposition, approximation coefficients and detailed coefficients are generated. Using these coefficients the energy parameters are to be calculated and compared with the reference signal in order to find out the features of the disturbance signal.

\section{HARDWARE RESULTS}

The hardware required for proposed work are laboratory setup for sag creation, level shifter circuit for positive voltage clamping, mbed controller for real time data acquisition and Central Server (PC) for storing and manipulating the signal for feature extraction.

In Central Station the GUI is designed using Visual C Sharp 2010. The real time samples from the controller are automatically saved into an excel file. The GUI has the options of visualizing the voltage signal in real time. The processing part is done by MATLAB application which is running as a background worker in the GUI. The results are displayed in the textbox and the variation can be projected on a progress bar in the GUI. The screenshot of the GUI is shown in Fig.7 and photograph of the experimental setup in Fig.8.

The MATLAB application processes the signal using DWTMRA. The signal reconstructed with inverse Wavelet transform is compared with reference signal for calculating the duration and magnitude of the voltage sag. The real time plotting of serial data coming from the controller is done by GUI and results can be saved in a text file using save button in the application.

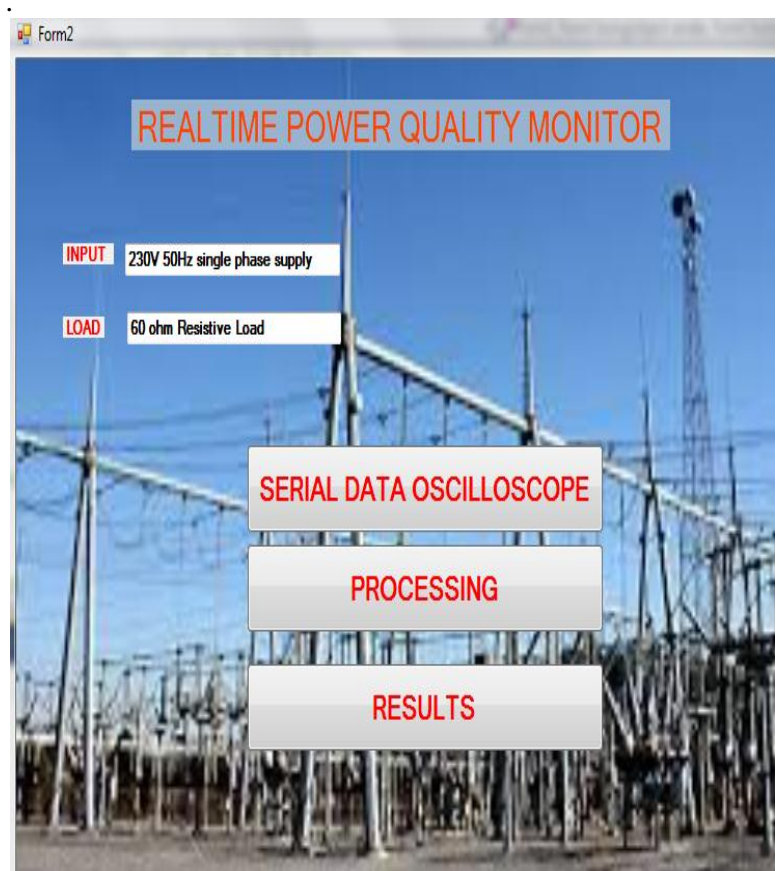

Fig 7: PQ Monitor Application

The experimental setup is created with $135 \mathrm{~V} 50 \mathrm{~Hz} 1 \phi$ ac supply and $15 \%$ - $20 \%$ sag is created using injection transformer for 3 seconds. The PQ monitor plots the real time serial data from the controller in the GUI. The application processes the given signal using DWT-MRA. The results obtained after processing is displayed in the textbox and the variation (\% of sag) is shown in the progress bar. The parameters of the sag obtained using automatic PQ analyzer are $111.719 \mathrm{Vrms}$ and 3.3 seconds. The Fig.9 provides the 


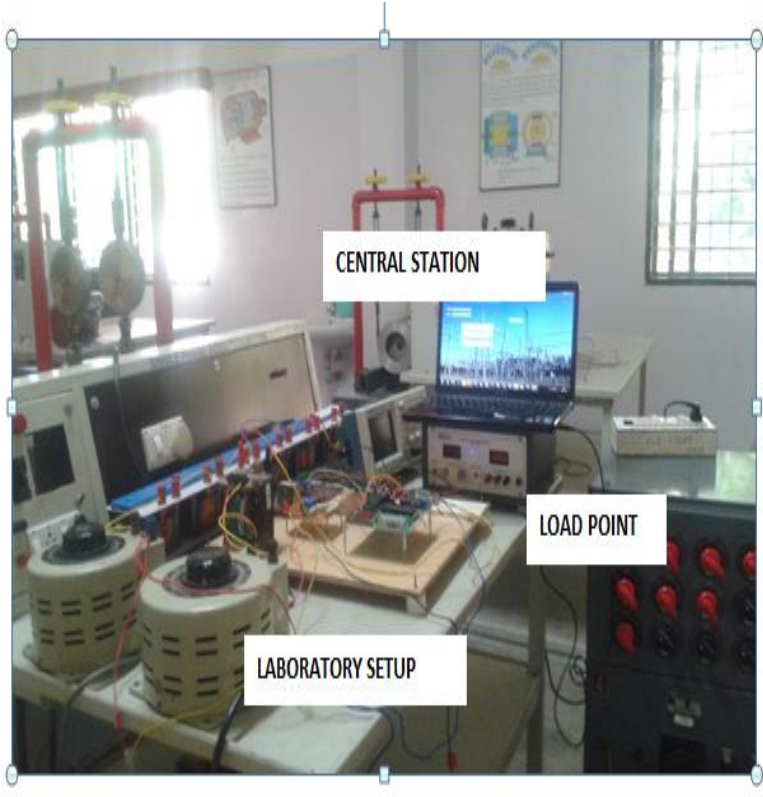

Fig 8: Photograph of Hardware Setup

screen shot of the serial data oscilloscope. The level shifted output voltage of $380 \mathrm{Vp}-\mathrm{p}$ is scaled down by 2 and displayed as 190Vp-p in the real time serial data oscilloscope. The $x$ axis represents the time in $\mathrm{ms}$ and $\mathrm{y}$ axis gives the peak to peak voltage. Fig.10 presents the results such as duration, magnitude and type of sag.

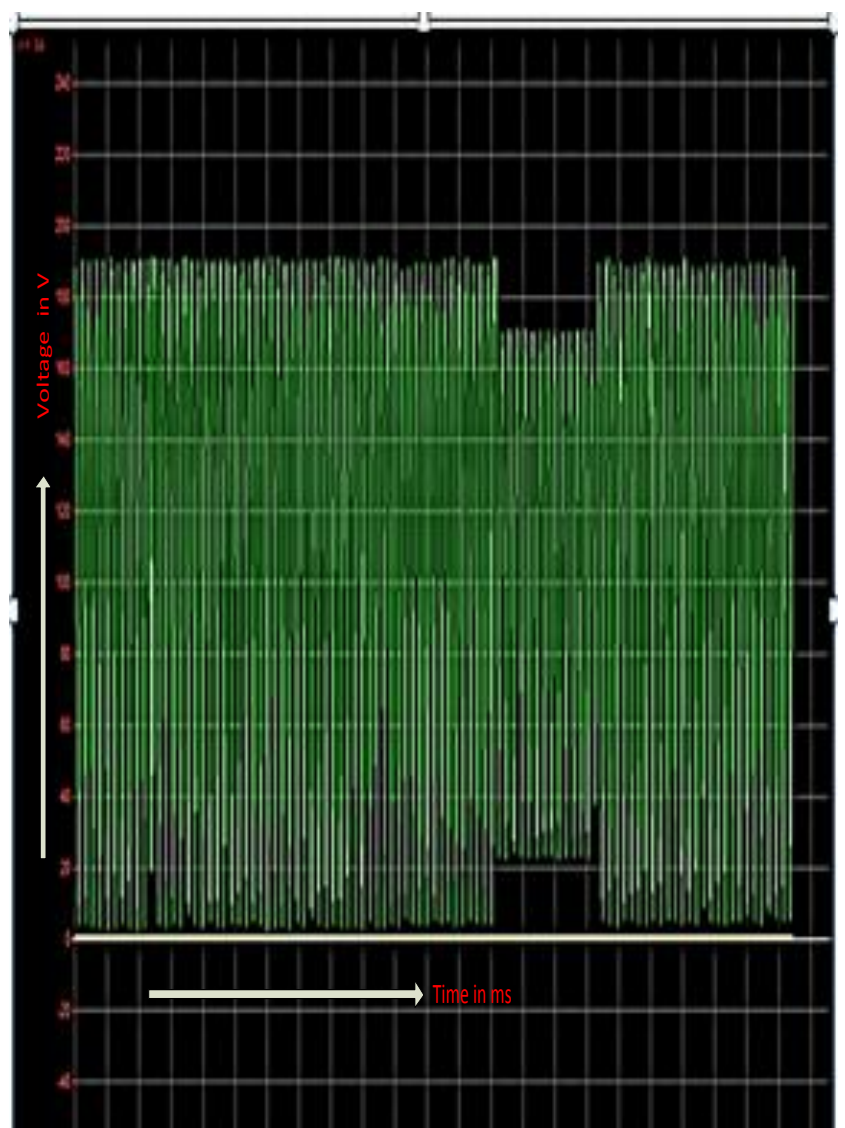

Fig 9: Photograph of GUI at the real time acquisition of Voltage Sag

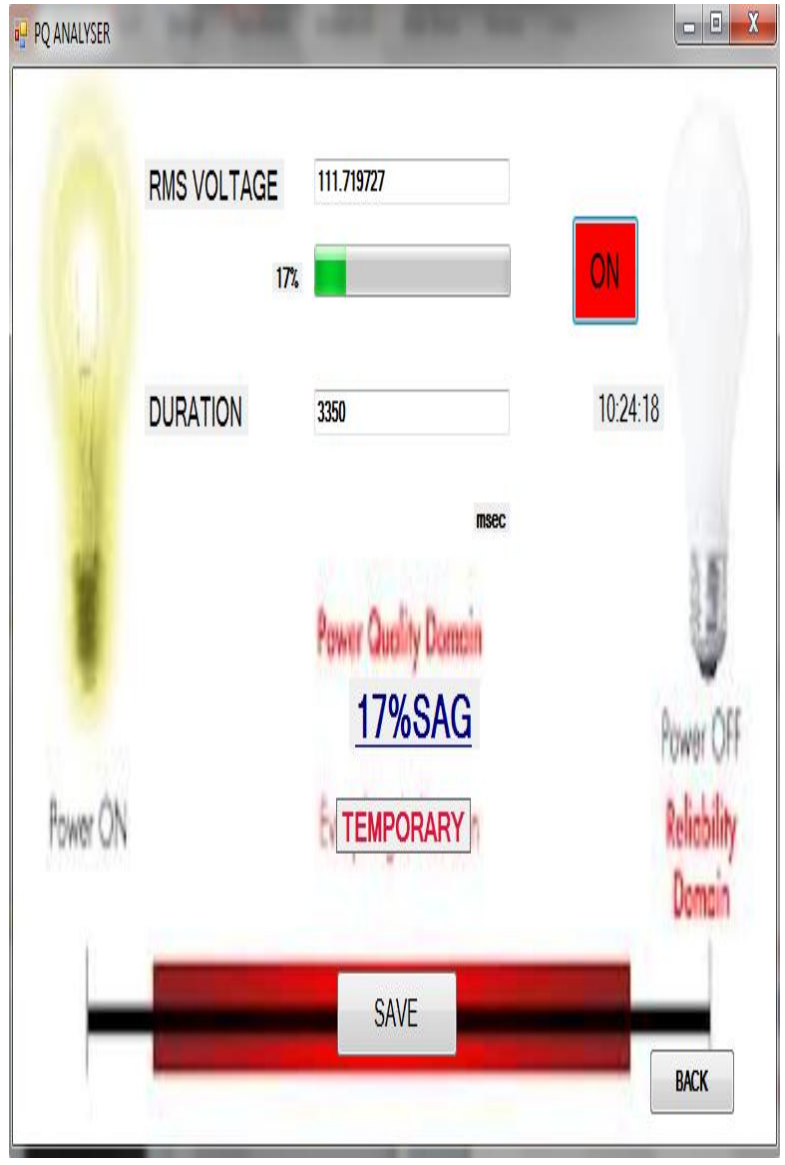

Fig 10: Snapshot of GUI displaying Results

\section{CONCLUSIONS}

In this paper a low cost embedded system for the detection of voltage sag is implemented. The laboratory setup for voltage sag is created using voltage injection method. The signal processing tool for the proposed work is Discrete Wavelet Transform with Multi Resolution Analysis. The results calculated using automatic processing is almost equal to the manual method of voltage sag recording with existing techniques. The proposed work provides a low cost intelligent power quality disturbance monitoring system making use of the capabilities of Wavelet Transform. The real time visualization and display of results are provided by GUI created using Visual C Sharp 2010

The proposed work can be extended by taking the real time scenario for the cause of voltage sag such as inductor motor starting, short circuits, three phase faults. .The control software logic can be modified to classify different PQ disturbances with the help of Artificial Neural Network with DWT. 


\section{REFERENCES}

[1] M. H. J. Bollen and I. Y. H. Gu, "Signal Processing of Power Quality Disturbances", Piscataway, NJ: IEEE Press, 2006.

[2] Yobo Duan, Xuhang Guo, "Research and design of power quality monitoring equipment", International Conference on Measurement, Information and Control (MIC), Vol.2, May 18-20 2012.

[3] Krisda Yingkayun, Suttichai Premrudeepreechacharn1, Neville R. Watson and Kohji Higuchi, "Power quality monitoring system based on embedded system with network monitoring", Scientific Research and Essays Vol. 7(11), pp. 1280-1292, 23 March, 2012.

[4] S. D. Pardeshi, M. Ramamooorty, R. B. Kelkar,"On-Line Measurement of Power Quality Parameters Using Signal Processing Techniques", Proceedings of the 7th WSEAS International Conference on Power Systems (PE'07), Beijing,China, September 2007, pp. 282-287.

[5] S.Suja, Jovitha Jerome, "Power Signal Disturbance Classification Using Wavelet Based Neural Network", Serbian journal of electrical engineering, Vol. 4, No. 1, June 2007, 71-83.

[6] J. Liu, P. Pilay, "Application of Wavelet Analysis in Power System Disturbance Modeling", IEEE Trans. AFRICON, Vol.2,No.X, 1999, pp. 639-642.

[7] M. Uyar, S. Yıldırım, M. T. Gençoğlu, "An Effective Wavelet-Based Feature Extraction Method for Classification of Power Quality Disturbance Signals", Elsevier Electric Power Systems Research, Vol.78, 2008, pp. $1747-1755$.
[8] M Karimi "Wavelet based on-line disturbance detection for power quality applications",IEEE Transactions on Power Delivery,2000,15(4):1212-1220.

[9] D. Saxena, S.N. Singh and K.S. Verma, "Wavelet based denoising of power quality events for characterization", International Journal of Engineering, Science and Technology Vol. 3, No. 3, 2011, pp. 119-132.

[10] J. Xargayó, J. Meléndez, J. Colomer, “Analysis strategy based on Wavelet decomposition for classification of Voltage sags", Control Engineering and Intelligent Systems Group - Institute of Informatics and Applications.

[11] Frederico Ramos Cesário,"Errors produced by method of detection of Voltage sag", ABCM Symposium Seriesi n Mechatronics - Vol. 5,Section VI Metrology, pp.913-920.

[12] S. Debdas, S. Paikra, and T. Roy Choudhury, Member, IACSIT, "Voltage sag Detection through Wavelet Energy Coefficient", International Journal of Computer and Electrical Engineering, Vol. 5, No. 4, pp.383386, August 2013.

[13] F. B. Costay,A. Souza and N. S. D. Brito, "Real-Time Detection of Voltage sags Based on Wavelet Transform", Transmission and Distribution Conference and Exposition: Latin America (T\&D-LA), 2010 IEEE/PES.

[14] Vasanthi V, Ashok S "Analysis of Harmonics of Electric Traction System using Wavelet Transform", International Journal of Computer Applications (0975 8887) Vol.71- No.24, June 2013 